\title{
Novel cardiovascular biomarkers and implications for clinical trials
}

\author{
Valentin Fuster
}

Risk stratification for cardiovascular disease continues to be one of the main research focuses in 2006. Despite the excitement surrounding evolving chemical, genetic and progenitor, and imaging markers, the so-called traditional risk factors, among them obesity, sedentary lifestyle and diabetes, will probably still account for more than $90 \%$ of cardiovascular events, or so-called hard endpoints, in the next decade. Yet, the presence of traditional risk factors does not necessarily imply a cardiovascular event will occur, and not all events will imply the presence of these risk factors. Thus, can surrogate endpoints, such as biomarkers or noninvasive imaging, improve prediction of cardiovascular events?

Among the chemical markers, two seem to have prognostic merit. The first, C-reactive protein (CRP) is a marker of atherothrombotic or systemic inflammation or both. In the US, it seems likely to improve the predictive value of traditional risk factors, such as the wide-range intermediate Framingham risk score (5-20\% at 10 years), not least because it can be measured rapidly by a low-cost, standardized assay. Whether in the US the predictive specificity of CRP matches its high sensitivity and whether it will prove as sensitive for populations outside the US require further investigation. The second chemical marker, known as brain natriuretic peptide (BNP), reliably indicates hemodynamic and ischemic myocardial stress. BNP is also entering the clinical arena, not only because of its predictive value but also its usefulness in the monitoring of therapeutic effectiveness in cardiac failure. Although CRP is evolving as a valuable addition to traditional risk assessment for atherothrombotic disease, and BNP is proving useful as a diagnostic and therapeutic monitoring tool, it appears that neither will reach the status of being a surrogate endpoint for cardiovascular events; no other genetic biomarkers are even so far advanced in status.
If premature

vascular

disease can

be observed

and quantified

before

cardiovascular

events occur

and therapeutic

response can

be monitored,

we will be

as close as

possible to

prediction and

prevention

$V$ Fuster is the Editorin-Chief of Nature

Clinical Practice

Cardiovascular

Medicine

\section{Competing interests}

The author declared he has no competing interests.

www.nature.com/clinicalpractice doi:10.1038/ncpcardio0477
Thus, most of cardiovascular diseases are complex in etiology, and many genetic polymorphisms thought to be linked to coronary artery disease or myocardial infarction have never been confirmed. By contrast, progenitor markers, such as bone-marrow cells, might eventually become relevant as biomarkers for vascular processes and cardiovascular events. For example, in animal models, we and others have demonstrated accelerated endothelial repair via bone-marrow-cell differentiation and progenitor-cell release. In humans, high blood levels of $\mathrm{CD} 34^{+} / \mathrm{KDR}^{+}$ progenitor cells have been associated with a decrease in cardiovascular event rates, whereas low levels, which imply endothelial damage, have been associated with a rise in cardiovascular events.

Perhaps some of the evolving noninvasive imaging technologies offer better hope for surrogate endpoints. If premature vascular disease can be observed and quantified before cardiovascular events occur and therapeutic response can be monitored, we will be as close as possible to prediction and prevention. For example, black-blood MRI sequences have been shown to quantify carotid and aortic premature vascular disease in a handful of individuals, and to demonstrate regression after the use of lipid-modifying strategies. Furthermore, complete luminal coronary imaging has been achieved with three-dimensional MRI in one acquisition of less than 30 min duration, with no radiation, and with diagnostic accuracy of 80-90\% for substantial coronary stenoses.

In summary, certain novel biomarkers and noninvasive imaging modalities might turn out to be highly sensitive, specific and reproducible in nearly all patients with cardiovascular disease. If so, they could be used to prioritize, simplify and modulate expensive clinical prevention trials of hard cardiovascular endpoints involving thousands of patients. 